\title{
The effects of quantity, complexity, and attentional demand on children's time perception
}

\author{
MARSHALL ARLIN \\ University of British Columbia, Vancouver, British Columbia, Canada
}

\begin{abstract}
An experiment was conducted to investigate the effects of quantity, complexity, and attentional demand on children's time perception. The appropriateness for children of two models of time perception founded primarily on adult research was examined. These models were the attentional hypothesis and the storage size hypothesis. Ninety-six children, 32 each of 6, 9, and 12 years, observed two and four displays of simple and complex shapes under conditions of higher (matching) or lower (looking) attentional demand. In accord with the attentional model, an inverse relationship was found between attentional demand and children's time perception. However, in accord with the storage size model, a positive relation was found between quantity and children's time perception. A developmental trend was also found: younger children were more susceptible to the quantity effect, and older children were more susceptible to the attentional demand effect. Results generalize adult findings to younger children, but the pattern of results is not easily explained solely by either model. Further refinements of either model, or development of a new model that can integrate the two separate findings, appears warranted.
\end{abstract}

Two models of time perception which have attracted considerable research attention are the attentional hypothesis and the storage size hypothesis. Much of the research cited in support of either model has been based on adult samples. The purpose of the present study was to examine the degree to which the predictions of either model could be extended to children's time perception. The focus of the present study was on three variables used in the adult research: quantity, complexity, and attentional demand.

\section{Quantity}

According to the storage size hypothesis, greater quantity presented during an interval will increase storage size and, as a result, will increase remembered duration. Using the retrospective paradigm, in which subjects are not told in advance that they will be asked about time, several researchers have confirmed this relationship between quantity and remembered duration (Block, 1974; McClain, 1983; Mulligan \& Schiffman, 1979; Ornstein, 1969). Other findings consistent with the storage size predictions for quantity are those in which filled intervals were perceived as longer than empty intervals (Buffardi, 1971; Mo, 1971, 1975; Thomas \& Brown, 1974).

I thank Nusri Kassam, Lee Johnston, and Brian Shaw for their help in testing the children. I thank the Social Sciences and Humanities Research Council of Canada (SSHRC) for funding which made part of this study possible. Finally, I thank the staff of Crescent Park School for their kind cooperation. Requests for reprints should be sent to Marshall Arlin, Department of Educational Psychology and Special Education, 2125 Main Mall, University of British Columbia, Vancouver, B. C., Canada V6T 1 Z5.
Extrapolating from the attentional hypothesis, quantity in itself is neither positively nor negatively related to perceived duration, but depends on intervening processes. If increased quantity causes increased attention to stimuli, as hypothesized for complexity, then the relationship between quantity and perceived time would be inverse: more quantity, more attention to stimuli, less attention for the timer, and shorter perceived duration. An important aspect of quantity is the quantity of perceived changes (Fraisse, $1963,1981,1982$ ) in an event or set of stimuli. To the extent that the perception of change requires attention, the attentional hypothesis would predict an inverse relationship between quantity and perceived time.

A variable closely related to quantity is frequency or presentation rate. Models of time perception based on the number of changes observed (Fraisse, 1961; Fraisse \& Zuili, 1966; Frankenhaeuser, 1959) often use presentation rate, or frequency, to manipulate perceived changes. These researchers posit a direct relationship between quantity as frequency and time perception. In the present study, quantity is understood in Fraisse's sense as quantity of perceived changes, and it was manipulated by varying presentation rate across a fixed interval.

\section{Complexity}

According to the storage size hypothesis (Ornstein, 1969), the experience of an interval's duration in retrospect is positively related to stimulus complexity. This retrospective experience, or "remembered duration" (Block, 1978), is a function of the memory storage size experienced during the interval. As complexity of stimulus events increases, one or more of the processes of coding, storage, or retrieval increases, storage size is in- 
creased, and consequently remembered duration is increased. A limited amount of evidence supports the storage size prediction about complexity (Block, 1978, Experiment 2; Ornstein, 1969). But a greater number of experiments have failed to replicate this prediction (Block, 1974, Experiment 2, 1978, Experiment 1; Bobko, Schiffman, Castino, \& Chiapetta, 1977; Kowal, 1976; Smith, 1975; Yeager, 1969).

The attentional model of Hicks, Miller, and Kinsbourne (1976) and Hicks, Miller, Gaes, and Bierman (1977) would predict the opposite pattern for complexity. Here I extrapolate from the model because, to my knowledge, attentional researchers have not manipulated complexity variables. According to this model, the degree of attention to time in passing by a metaphorical cognitive timer is inversely related to perceived and remembered duration. When attention is drawn away from the timer by task demands, less attention is paid to time in passing, and time seems briefer (Fraisse, 1963, pp. 145-147, 201210 ). It seems plausible that complex stimuli might engage attention to a greater degree than simple stimuli. Consequently, less attention would be directed to the cognitive timer, and duration would seem briefer for an interval containing complex stimuli than for an interval with simple stimuli.

\section{Attentional Demand}

The storage size hypothesis does not appear to predict effects for attentional demand, other than when attentional demand is confounded with such factors as quantity.

The attentional hypothesis predicts an inverse relationship between attentional demand and time perception. Greater attentional demand is associated with less perceived time. Within an attentional demand framework, tasks requiring active attention will distract attention from the cognitive timer, resulting in briefer time perception. In contrast, for tasks with fewer attentional demands, attention will be left over to monitor time in passing, resulting in increased time perception. A number of studies have found decreased time perception under conditions requiring active attention (Burnside, 1971; Curton \& Lordahl, 1974; Devane, 1974; Hicks \& Brundige, 1974; Hicks et al., 1977; McKay, 1977; Zakay, Nitzan, \& Glicksohn, 1983).

In much of the research cited previously for quantity, the operational measurement of quantity, one which serves as a proxy for storage size, is input quantity. Extending the previous line of reasoning from quantity as an input measure to quantity as an output measure, attentional demand could be a confounding factor that can explain findings of both a positive and a negative relationship between quantity and perceived time. For example, the output from an interval might be greater as a result of deeper processing or greater attention, resulting in the inverse relationship between quantity and perceived time (more output, less time). Conversely, attentional demand could also explain a positive relationship between quantity and perceived time if greater attentional demand or greater depth of processing were associated with fewer, more difficult stimuli, as in Burnside's (1971) mathematical tasks (less output, less time). By the factorial combination of quantity and attention in the present study, the potential confounding should be disentangled.

To summarize, the purpose of the present study was to extend the predominantly adult research cited above to children, and to determine the extent to which either of the two models provides a valid explanation of the effects (if any) of quantity, complexity, and attentional demand on young children's perception of time.

\section{METHOD}

\section{Subjects}

Participants in the study were 96 children, 32 each in kindergarten and 3rd and 6th grades. The children were sampled randomly from nine classrooms in a school from a middle to upper middle SES neighborhood in a suburb of Vancouver, Canada. Five intellectually handicapped children were not included in the sampling base. The children were tested in the second half of the school year, with the children from all three levels being interspersed across each of the months of testing. Age in months at the time of testing was recorded, and the mean ages were converted to years as follows: $\mathrm{K}=5.9$ years; 3 rd $=8.9$ years; 6 th $=12.1$ years.

\section{Materials}

The materials were six sets of simple figures and six sets of complex figures generated on 12 computer screens. Each screen contained two similar figures, one at the top and one at the bottom. Horizontal position of the top and bottom figures was varied randomly. Each figure was made from nine oversize, uppercase letters (twice the width and height of standard uppercase). Only one letter, repeated nine times, was used in each figure, and the same letter was used for both figures in a screen. The six letters used were: $\mathrm{X}, \mathrm{O}, \mathrm{Y}, \mathrm{H}, \mathrm{Z}, \mathrm{U}$. Simple figures comprised a single line with one break of two spaces, as shown in Figure 1. Complex figures had three levels, nine spaces wide, with the nine letters alternating up and down among the three levels, also shown in Figure 1.

As a check on the degree to which the figures used did represent differences in stimulus complexity, the following scaling procedure was used. Nine undergraduate and two graduate students enrolled in Educational Psychology courses at the University of British Columbia were asked to rank one figure from each of the 6 simple shapes and one from each of the 6 complex shapes. The resulting 12 shapes were randomly interspersed, and subjects ranked all from most simple (rank 1) to most complex (rank 12). They were given the following definitions adapted from Webster's second college edition of the American Heritage Dictionary: "The word COMPLEX is used to describe a thing having many parts so interconnected that the whole thing is difficult to understand. The word SIMPLE is used to describe a thing that is not involved or complicated, and without additions or modifications." Then the ranking instructions were given.

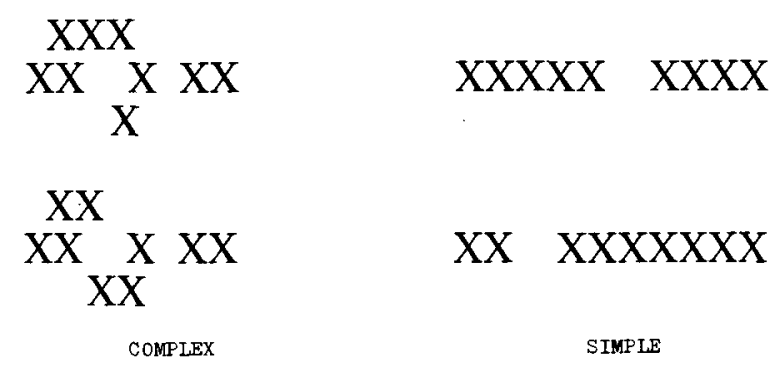

Figure 1. Examples of complex and simple screens. 
Assuming a perfect correspondence to the experimenter's dichotomy, the lowest possible mean score for the aggregate of the six simple shapes would be 3.5 ; and the highest possible mean ranking for the aggregate of the six complex shapes would be 9.5 . The actual mean aggregate rankings were 3.5 for the simple figures and 9.5 for the complex figures. The means and standard deviations for each of the six simple figures were: $4.09(1.38), 3.36(1.50)$, $3.36(1.69), 4.00(1.41), 1.64(1.21)$, and $4.54(1.73)$. Those for the six complex figures were: $9.91(1.81), 9.64(1.63), 8.73$ (1.85), 9.82 (1.47), 8.54 (1.29), and $10.36(1.80)$.

Another way of representing the results is to score any ranking of a simple figure from 1 to 6 and of a complex figure from 7 to 12 as an agreement, and any other rankings as a disagreement. On this basis, the percentage agreement was $100 \%$, indicating interjudge agreement with the categorization of figures into simple and complex according to the operational definition used above.

Screens were combined into sets, with one simple set containing two screens (four figures) and one complex set also containing two screens. In each of these sets, one screen was made of Xs and the other Os. Two other sets, one simple and one complex, contained four screens (eight figures) and used the letters $Y, H, Z$, and $U$, one per screen.

\section{Procedure}

At each grade level, 16 pupils were assigned, by a table of random numbers, to the higher attentional demand condition (matching) and 16 pupils were assigned to the lower attentional demand condition (looking). The pupils were tested individually in a room outside their classroom. The experimenter used an Apple Ile computer with a green monochrome monitor and a printed circuit board clock for timing.

For the first warm-up, the children were shown a computer key that produced continuous tones, and they practiced pressing it. Then they were told that they would hear tones generated by the computer, after which they would hold down the key for the same length of time as each tone. The computer then generated three tones in a sequence of 2,10 , and $5 \mathrm{sec}$, after each of which the child responded for the perceived equivalent time. If the child was confused about the task, feedback was provided and the warm-up was repeated. For a child to be included in the experiment, it was necessary that the rank order of his or her reproductions correspond to that of the stimulus tones. One kindergarten child and one third grade child were replaced because of difficulty with the first warm-up.

The second warm-up used three $81 / 2 \times 11$ in. sheets of paper with simple figures on the top and bottom of two of them and with complex figures on one. All the figures contained four uppercase letters, rather than the nine letters of the experimental stimuli. In the lower demand condition (looking), the children were told, "You're going to see pictures like this on the computer screen. Afterwards, you will press the button down for as long as all the pictures were on the screen." Then the three sheets of figures were shown in sequence.

In the higher attentional demand condition (matching), the children were told "You're going to see pictures like this one and you'll have to decide whether the picture on the top is the same or different as the one at the bottom. Now we'll practice on three of them." The children attempted all three, and then were given feedback about their performance. The children were asked in what way the figures were the same or different, and if they could not explain, the experimenter pointed out the differences or reviewed the similarities. Horizontal placement was random on the warm-up as well, so the experimenter pointed out to the children that "it doesn't matter if the pictures are more on this side or on that side." One kindergarten child in the higher attentional demand condition could not understand the matching task and had to be replaced.
Each child observed four sets of screens, accompanied by a brief tone at the beginning and termination of each set. The duration of each set of screens was $12 \mathrm{sec}$. The four sets were: (1) two simple screens at 6 sec each, (2) four simple screens at $3 \mathrm{sec}$ each, (3) two complex screens at $6 \mathrm{sec}$ each, and (4) four complex screens at $3 \mathrm{sec}$ each. After each set of screens, the children reproduced the estimated time by pressing the computer button. Reproduction scores were recorded in hundredths of a second. Using a Latin squares procedure, the children were assigned to one of four sequences, each beginning with a different set.

\section{RESULTS}

One child's set of scores was incomplete, so the final sample for analysis was 95 . As a check on the degree to which the 48 children (16 per grade) in the higher attentional demand condition were actually following the matching directions, an examination of the accuracy scores was conducted. For the easiest choice, matching two simple objects, the proportions of correct responses were $91 \%, 97 \%$, and $100 \%$, respectively, for grades K, 3 , and 6; for four simple matches, the proportions were $81 \%, 98 \%$, and $88 \%$. (The drop at the 6 th grade appears to have been due to some 6 th grade students' thinking the task was more difficult and therefore not producing a response.) For two complex matches, the proportions were $82 \%, 94 \%$, and $97 \%$. The above all indicate that pupils were attempting to match the pictures, and the responses at all grades were considerably above a chance level of $50 \%$. The only condition in which the responses approached the chance level was that of four complex matches, for which the proportions were $47 \%, 69 \%$, and $80 \%$, respectively, for grades $K, 3$, and 6 . But even these results reflect high attention to the task. Many of the errors were due to nonproduction of an answer (in lieu of guessing) because, as many children said, "the pictures came too fast." On the basis of these results, it seems reasonable to conclude that the higher attentional demand condition did indeed represent a condition of attention through matching.

A preliminary analysis of reproduction scores indicated that neither sex nor sequence were significant $(F \mathrm{~s}<1)$, but that there was an interaction of sequence $X$ the four trials $[F(9,261)=3.3, p<.001]$. Sets appearing first were reproduced as shorter than sets appearing in other positions.

Reproduction scores were collapsed across sex and sequence, and the resultant means, by quantity, complexity, attentional demand, and grade, are shown in Table 1.

A four-factor split-plot analysis of variance was performed on the reproduction scores. The between-group factors were grade level $(K, 3,6)$ and attentional demand (greater, lesser). The within-subject factors were complexity (simple, complex) and quantity (two screens, four screens). There were significant main effects for age $[F(2,89)=17.0, p<.001]$, for attentional demand $[F(1,89)=44.1, p<.001]$, and for quantity $[F(1,89)$ $=40.3, p<.001]$. Complexity was not significant. All 
Table 1

Mean Time Reproduction as a Function of Attentional Demand, Complexity, and Quantity: By Grade

\begin{tabular}{cccccc}
\hline \multirow{2}{*}{$\begin{array}{c}\text { Number } \\
\text { of Screens }\end{array}$} & \multicolumn{2}{c}{ Low Attention } & & \multicolumn{2}{c}{ High Attention } \\
\cline { 2 - 3 } \cline { 5 - 5 } & Simple & Complex & & Simple & Complex \\
\hline & \multicolumn{5}{c}{ Kindergarten } \\
4 & 299 & 382 & 293 & 330 \\
& 569 & 602 & 445 & 413 \\
2 & & Grade 3 & & \\
4 & 882 & 864 & 405 & 375 \\
& 906 & 901 & 452 & 494 \\
2 & & Grade 6 & & \\
4 & 923 & 843 & 560 & 473 \\
& 1069 & 1014 & 635 & 518 \\
\hline
\end{tabular}

Note-Times are in hundredths of a second.

children reproduced times that were significantly shorter than the 12-sec standard, but those of the kindergarten children were considerably shorter $(4.16 \mathrm{sec})$ than those of the other two grades $(6.60$ and $7.54 \mathrm{sec})$. Intervals with greater attentional demand were perceived as being significantly shorter $(4.49 \mathrm{sec})$ than those with lesser attentional demand $(7.71 \mathrm{sec})$. And two screens were perceived as being shorter $(5.52 \mathrm{sec})$ than four screens $(6.68 \mathrm{sec})$.

Interactions were tested by treatment $x$ trend-contrast planned comparisons. The interaction was decomposed orthogonally into the linear and quadratic grade $\times$ depth complex contrasts (Kirk, 1982). The attentional demand $\times$ linear contrast interaction was significant $[F(1,89)=$ $7.3, p=.008]$, as was the attentional demand $\times$ quadratic contrast $[F(1,89)=3.9, p=.047]$. The differential effect of processing (seconds looking minus seconds matching) was much greater for older children, with a difference of 4.57 and $4.15 \mathrm{sec}$ for grades 3 and 6 and only .93 sec for the kindergarten children. Post hoc orthogonal decompositions of the interaction were then conducted and tested by the Dunn estimated $F$. All of the interaction was due to the difference between the combined effects for 3rd and 6th grade children versus the kindergarten children $[F(1,89)=11.0, p<.002]$. There was no difference between the effects of attentional demand on 3rd grade versus 6 th grade children $(F<1)$.

The same contrast vectors were used to examine the linear and quadratic quantity $\times$ trend-contrast interactions. The linear, a priori, complex comparison was not significant $[F(1,89)=2.6, p>.05]$. But the quadratic interaction component was significant because of the 3rd grade scores $[F(1,89)=5.3, p=.022]$. Post hoc orthogonal decompositions of the interaction were then conducted, using Dunn's estimated $F$. The quantity effect was significantly greater for kindergarten children than for the other two grades $[F(1,89)=6.4, p=.013]$, but there was no differential effect between grades 3 and $6[F(1,89)$ $=1.4, p>.05]$. Kindergarten children were more susceptible to the quantity effect (seconds 4 shapes minus seconds 2 shapes), taking $1.83 \mathrm{sec}$ longer for the larger quantity; for grades 3 and 6 , the quantity effect was only .56 and $1.09 \mathrm{sec}$. In both of these interactions, the treatment-contrast interactions were not strictly linear, as hypothesized, but contained a quadratic component because the 3 rd grade scores were anomalous, either above or below the linear interaction trend line.

\section{DISCUSSION}

The combined findings of a significant effect for attentional demand and no effect for stimulus complexity generalizes aspects of the attentional model (Hicks et al., 1976) to young children's time perception. Moreover, the finding of a significant effect of quantity generalizes aspects of the storage size hypothesis (Ornstein, 1969) to young children's time perception.

Children at all ages of this sample (particularly 9 and 12 years) reproduced shorter durations after tasks with greater attentional demand than after tasks with lesser attentional demand. This finding extends adult studies that support attentional models (Burnside, 1971; Curton \& Lordahl, 1974; Devane, 1974; Hicks \& Brundige, 1974; Hicks et al., 1977; McKay, 1977; Zakay et al., 1983) to younger populations. This effect was not only significant, but was also quite large, with low-attention intervals reproduced as $72 \%$ longer than higher attention intervals. It appears that children as young as 9 and 12 years are as sensitive to attentional manipulations as many of the adults in the studies cited above.

Furthermore, the present results suggest a developmental trend for the effects of attentional demand. Kindergarten children were less influenced by attentional differences than 3rd and 6th graders. This finding is consistent with a recent finding by Arlin (1986) of a linear treatment $x$ trend contrast interaction of depth of processing $x$ age. In Experiment 2 of that study, children categorized pictures as either living or nonliving (deep) or repeated the experimenter-provided label (shallow). From almost no effect of depth, the effect increased steadily across the grade levels $\mathrm{K}, 2,4$, and 6 . It may be that young children's attention is less differentially activated than older children's, and is more of an either-or process. Perhaps many tasks that adults consider deep versus shallow or high versus low attention fully (or at least extensively) engage children's attention, thereby lessening an effect on time perception. Further research is needed to determine if other attentional manipulations are more suitable to younger (5-7 years) children. Moreover, because of the instability of interactions, further research is needed to determine the extent to which the developmental trends in the Arlin (1986) study and the present one can be generalized.

The failure to find the effect for complexity predicted by the storage size model supports and extends downward adult studies (Block, 1974, Experiment 2, 1978, Experiment 1; Bobko et al., 1977; Kowal, 1976; Smith, 1975; Yeager, 1969). The complexity findings in support of the storage model (Block, 1978, Experiment 2; Ornstein, 1969) may be limited to specific, primarily retrospective conditions, and to samples older than the age range of 
the present study. Young children's processing or storage may be much less easily activated than that of adults. Adults' self-generated processing or storage might be activated by stimulus differences between simple and complex figures, but children might remain passive toward both without explicit processing, attentional, or storage directions. Given explicit processing or attentional directions, the predicted effect might be found, as it was in the present study.

A related explanation of the null complexity results might be that the complex stimuli were above the channel capacity of the young children in the study. The excess information might not have registered and, consequently, the complex figures would have been equivalent to the simple figures. This explanation could also be used to explain the lack of attentional demand effect for the very young children. Ornstein (1969, Experiment 2) used this argument (but for retrospective comparisons) when he found a differential effect between simple and moderately complex stimuli but not among successively more complex stimuli.

But even if the channel-capacity explanation is appropriate, it is hard to see it as confirming the storage size hypothesis. Even Ornstein (1969, p. 63) felt that his complexity result only "provisionally" confirmed the storage size hypothesis, and "with some difficulties." For complexity results to support the storage size hypothesis for children, it would seem necessary for levels of complexity within children's channel capacity to activate storage size differentially through coding, storage, or retrieval processes. But, unless the storage processes are independent from attentional processes, the results are likely to be similar to those found above, suggesting some boundaries to the generalizability of the storage size hypothesis.

The finding of a positive relationship between quantity and time reproduction is consistent with predictions and findings in support of the storage size hypothesis (Block, 1974; McClain, 1983; Mulligan \& Schiffman, 1979; Ornstein, 1969). It also supports researchers who stress the positive relationship between time perception and quantity of perceived changes (Fraisse, 1961, 1981, 1982; Fraisse \& Zuili, 1966; Frankenhaeuser, 1959; Zuili \& Fraisse, 1966) and those who have found filled intervals to be perceived as longer than empty intervals (Buffardi, 1971; Mo, 1971, 1975; Thomas \& Brown, 1974), although in the present study, lower quantity rather than empty quantity was used.

But, although this finding is consistent with storage size predictions, it does not provide unambiguous support of the storage size model. The variable label "quantity," rather than "storage size," has been used purposely in the present study to indicate a stimulus characteristic rather than a cognitive processing characteristic. The relationship between input quantity and storage quantity is plausible, but not necessary. Even Ornstein (1969) has used input quantity as a proxy for storage quantity, but no such claim is made here.
As an alternative to the storage size explanation, the quantity finding may reflect a learned probability that a greater number of events corresponds to greater time. Along a similar line of thought, the quantity finding supports Levin's "more $X$ is more time" hypothesis (Levin, 1977, 1979; Levin \& Gilat, 1983). According to Levin, children's comparative judgments are influenced by the sheer magnitude of cues, even irrelevant ones, so that more of any variable $(X)$ is equated with more time. For example, children may judge an interval containing a bright light to be longer than the same interval containing a dimmer light (more light $=$ more time). If Levin's hypothesis explains the present results, then her hypothesis is valid not only for the comparative judgments she used but also for reproduction, as used in the present study.

An interesting, but tenuous, finding about quantity was its interaction with age. Young children were most susceptible to its influence. Presumably, young children are less able to disengage other cues, such as quantity, from the experience of time in passing (or at least from the reproduction of that experience). The curvilinear trend caused by the best performance (least influence from the quantity illusion) at 3rd grade is problematic. If this is not sampling or other random error, then an explanation of the developmental shifts and apparent regressions will be necessary.

In conclusion, some adult findings that have been cited in support of either the storage size or attentional model appear to generalize to children. However, neither model provides a comprehensive and consistent explanation of all the results. Rather, the attentional model explains the effects of attentional demand, and the storage size or perceived change model explains the effects of quantity. Further elaboration of the present models or development of new models appears warranted to integrate the effects of quantity and attentional demand on children's perception of time.

\section{REFERENCES}

ARLIN, M. (1986). The effects of quantity and depth of processing on children's time perception. Journal of Experimental Child Psychology, 42, 84-98.

BLOCK, R. A. (1974). Memory and the experience of duration in retrospect. Memory \& Cognition, 2, 153-160.

BLock, R. A. (1978). Remembered duration: Effects of event and sequence complexity. Memory \& Cognition, 6, 320-326.

Bobko, D. J., Schiffman, H. R., Castino, R. J., \& Chiapetta, W. (1977). Contextual effects in duration experience. American Journal of Psychology, 90, 577-586.

BUFFARDI, L. (1971). Factors affecting the filled-duration illusion in the auditory, tactual, and visual modalities. Perception \& Psychophysics, 10, 292-294.

BURNSIDE, W. (1971). Judgment of short time intervals while performing mathematical tasks. Perception \& Psychophysics, 9, 404-406.

Curton, E. D., \& LordahL, D. S. (1974). Effects of attentional focus and arousal on time estimation. Journal of Experimental Psychology, 103, 861-867.

DEVANE, J. R. (1974). Word characteristics and judged duration for two response sequences. Perceptual \& Motor Skills, 38, 525-526.

Fraisse, P. (1961). Influence de la durée et de la fréquence des change- 
ments sur l'estimation du temps [The effect of duration and frequency of changes on time estimation]. Année Psychologique, 61, 325-339. Fraisse, P. (1963). The psychology of time. New York: Harper \& Row. FraIsSE, P. (1981). Cognition of time in human activity. In G. D'Ydewalle \& W. Lens (Eds.), Cognition in human motivation and learning (pp. 233-259). Hillsdale, NJ: Erlbaum.

Fraisse, P. (1982). The adaptation of the child to time. In The developmental psychology of time (pp. 113-140). New York: Academic Press.

Fraisse, P., \&uILI, N. (1966). L'Estimation de la duré [The estimation of duration]. In Psychologie et épistémologie génétique. Themes Piagetiens (pp. 253-269). Paris: Dunod.

FrankenhaEuSER, M. (1959). The psychology of time. Stockholm: Almqvist \& Wiksell.

Hicks, R. E., \& Brundige, R. M. (1974). Judgements of temporal duration while processing verbal and physiognomic stimuli. Acta Psychologica, 38, 447-453.

Hicks, R. E., Miller, G. W., Gaes, G., \& Bierman, K. (1977). Concurrent processing demands and the experience of time-in-passing. American Journal of Psychology, 90, 431-446.

Hicks, R. E., Miller, G. W., \& Kinsbourne, M. (1976). Prospective and retrospective judgements of time as a function of amount of information processed. American Journal of Psychology, 89, 719-730.

KIRK, R. E. (1982). Experimental design: Procedures for the behavioral sciences (2nd ed., pp. 489-564). Belmont, CA: Brooks/Cole Publishing.

Kowal, K. H. (1976). Apparent duration of long meaningful events and meaningless intervals. Memory \& Cognition, 4, 215-220.

LEVIN, I. (1977). The development of time concepts in young children: Reasoning about duration. Child Development, 48, 435-444.

LEVIN, I. (1979). Interference of time-related and unrelated cues with duration comparisons of young children: Analysis of Piaget's formulation of the relation of time and speed. Child Development, 50, 469-477.

Levin, I., \& Gilat, I. (1983). A developmental analysis of early time concepts: The equivalence and additivity of the effect of interfering cues on duration comparisons of young children. Child Development, 54, 78-83.

MCCLAIN, L. (1983). Interval estimation: Effect of processing demands on prospective and retrospective reports. Perception \& Psychophysics, 34, $185-189$.

MCKAY, T. D. (1977). Time estimation: Effects of attentional focus and of a comparison of interval conditions. Perceptual \& Motor Skills, 45, 584-586.

Mo, S. S. (1971). Judgement of temporal duration as a function of numerosity. Psychonomic Science, 24, 71-72.

Mo, S. S. (1975). Temporal reproduction of an interval as a function of numerosity. Bulletin of the Psychonomic Society, 5, 165-167.

Mulligan, R. M., \& Schiffman, H. R. (1979). Temporal experience as a function of organization in memory. Bulletin of the Psychonomic Society, 14, 417-420.

ORNSTEIN, R. E. (1969). On the experience of time. Harmondsworth, England: Penguin.

Smith, M. J. (1975). Changes in judgement of duration with different patterns of auditory information for individuals confined to bed. Nursing Research, 24, 93-98.

Thomas, E. A. C., \& Brown, I., JR. (1974). Time perception and the filled-duration illusion. Perception \& Psychophysics, 16, 449-458.

YEAGER, J. (1969). Absolute time estimates as a function of complexity and interruption of melodies. Psychonomic Science, 15, 177-178.

ZaKaY, D., Nitzan, D., \&uicksohN, J. (1983). The influence of task difficulty and external tempo on subjective time estimation. Perception \& Psychophysics, 34, 451-456.

Zuili, N., \& Fraisse, P. (1966). L'estimation du temps en fonction de la quantité de mouvements effectives dans une tâche [Time estimation as a function of quantity of movements in a task]. L'Année Psychologique, 66, 383-396.

(Manuscript received January 24, 1986; revision accepted for publication July 7,1986 .) 